\title{
Force and location between direct and indirect laryngoscopes
}

\author{
Yukihiro Imajo $^{1}$ - Nobuyasu Komasawa ${ }^{1} \cdot$ Toshiaki Minami $^{1}$
}

Received: 25 August 2015 / Accepted: 9 December 2015 / Published online: 9 January 2016

(C) Japanese Society of Anesthesiologists 2016

To the Editor:

We compared the force required for laryngoscopy and location of applied force (LOF) between the Pentax-AWS Airwayscope $^{\circledR}$ (AWS; Hoya, Tokyo, Japan) and the Macintosh laryngoscope (McL) using simulation. On January 31 and July $5,2015,17$ anesthesiologists $(6.9 \pm 5.3$ years of clinical experience) were recruited. The Difficult Airway Management Simulator Evaluation System ${ }^{\circledR}$ (Kyotokagaku, Kyoto, Japan) was used to perform intubations on a manikin. Either a size $3 \mathrm{McL}$ blade or a standard AWS Intlock blade was used. During laryngoscopy and tracheal intubation, the force required for laryngoscopy and LOF was measured and calculated by the four sensors attached to the tongue of the simulator (Sup Fig a). The force required for laryngoscopy was calculated from the sum of the values in the four areas and LOF and was based on the formula shown in Sup Fig b. The results were compared using the Mann-Whitney $U$ test for force required for laryngoscopy, chi-squared test for Cormack-Lehane classification (CLC), and two-way repeated ANOVA for LOF. Data are presented as mean $\pm \mathrm{SD} . P<0.05$ was considered statistically significant. The order of the AWS and McL trial was randomized to minimize bias. The CLC did not differ significantly between McL and AWS (McL: 1 for 15, 2 for 2; AWS: 1 for 16,2 for $1, P=0.545$ ). The force required for laryngoscopy applied to the tongue was significantly less for the AWS than for the McL (AWS: $12.6 \pm 11.2 \mathrm{~N}$; McL: $18.2 \pm 5.7 \mathrm{~N}, P=0.016$ ) (Sup Fig c). The LOF also differed significantly-the AWS was significantly closer to the midline and deeper compared with the $\mathrm{McL}(X$ axis: AWS: $60.4 \pm 15.1 ; \mathrm{McL}: 87.3 \pm 6.2 \mathrm{~N}, P<0.001 ; Y$ axis: AWS: $109.2 \pm 5.5 \mathrm{~N}$; McL: $95.8 \pm 7.0 \mathrm{~N}, P<0.001$ ) (Sup Fig a, d, e). Our findings suggest that the force required for laryngoscopy is less with the AWS than with the McL, and that the LOF also differs by device.

\section{Compliance with ethical standards}

Conflict of interest None.

Electronic supplementary material The online version of this article (doi:10.1007/s00540-015-2131-8) contains supplementary material, which is available to authorized users.

Nobuyasu Komasawa

ane078@poh.osaka-med.ac.jp

1 Department of Anaesthesiology, Osaka Medical College, Daigaku-machi 2-7, Takatsuki, Osaka 569-8686, Japan 